\title{
Severe Non-Islet Cell Hypoglycemia From Ileum-Origin Gastrointestinal Stromal Tumor Producing Insulin-Like Growth Factor-2 in a Patient With Liver Cirrhosis Due to Chronic Hepatitis B
}

\author{
Kohzo Takebayashia, d, Sho Furukawa ${ }^{\mathrm{a}}$, Takenori Okumura ${ }^{\mathrm{a}}$, Mio Kubo ${ }^{\mathrm{a}}$, Atsushi Ujiie ${ }^{\mathrm{a}}$, \\ Mototaka Yamauchi ${ }^{a}$, Hiroyuki Shinozaki ${ }^{a}$, Kenji Hara ${ }^{a}$, Takafumi Tsuchiya ${ }^{a}$, Yuko Ono ${ }^{b}$, \\ Toshihiko Inukaic, Koshi Hashimoto ${ }^{a}$
}

\begin{abstract}
Non-islet cell tumor hypoglycemia (NICTH) is a rare paraneoplastic syndrome, and NICTH associated with gastrointestinal stromal tumor (GIST) is even more rare. Herein, we describe a patient with severe NICTH due to GIST who had developed liver cirrhosis as a consequence of chronic hepatitis B. Although circulating insulin, Cpeptide, and insulin-like growth factor-1 (IGF-1) levels were significantly decreased, in contrast to our expectations, the growth hormone (GH) level was slightly elevated. Steroid therapy with prednisolone appeared to be effective for the prevention of severe and continuous hypoglycemia.
\end{abstract}

Keywords: NICTH; GIST; Liver cirrhosis; Steroid therapy

\section{Introduction}

Non-islet cell tumor hypoglycemia (NICTH), which can frequently cause persistent hypoglycemia, is a rare paraneoplastic syndrome that was first reported in 1929, and that is mostly found in patients with epithelial and mesenchymal tumors [1-3]. In NICTH, high molecular weight insulin-like growth factor-2 (IGF-2), also known as big-IGF-2, is produced by in-

Manuscript submitted August 17, 2020, accepted September 5, 2020

Published online December 18, 2020

aDepartment of Diabetes, Endocrinology and Hematology, Dokkyo Saitama Medical Center, Koshigaya, Saitama, Japan

bDepartment of Pathology, Dokkyo Saitama Medical Center, Koshigaya, Saitama, Japan

'Department of Internal Medicine, Seibu General Hospital, Omiya, Saitama, Japan

${ }^{\mathrm{d} C}$ Corresponding Author: Kohzo Takebayashi, Department of Diabetes, Endocrinology and Hematology, Dokkyo Medical Center, Koshigaya, Saitama, Japan.Email: takeb@gmail.plala.or.jp

doi: https://doi.org/10.14740/jocmr4326 complete processing of pro-IGF-2 in the tumor cells, and this is thought as major cause of the hypoglycemia [4]. Usually normal IGF-2 forms inactive ternary complexes in the circulation, consisting of IGF-2, IGF binding protein (IGFBP)-3 and the acid labile subunit. On the other hand, big-IGF-2 cannot form the ternary complex and exists as a binary complex with IGFBP3 or free big-IGF-2, resulting in easy passing through the capillary membrane and probably easy binding to insulin receptor $[5,6]$. The activation of insulin receptors by IGF-2 can lead to hypoglycemia mainly by inhibiting glucose secretion, glycogenesis, and gluconeogenesis in the liver and by increasing glucose consumption in skeletal muscle $[5,7]$.

Gastrointestinal stromal tumor (GIST) is a common mesenchymal neoplasm in the gastrointestinal tract that is most often found in the stomach [8-10]. It is thought that GIST is derived from the intestinal cells of Cajal within the muscle layer which is associated with gastrointestinal motility $[8,11]$. GIST in most patients is involved in oncogenic mutations and overexpression of the tyrosine kinase receptor KIT [12]. Although GIST is included among the mesenchymal tumors, NICTH associated with GIST is very rare [13-16]; and to our knowledge there have been no previous reports of NICTH in patients with GIST complicated with liver cirrhosis, which may sometimes be the cause of hypoglycemia [17-19].

Herein, we report a case of a patient with both ileum-originating GIST and liver cirrhosis by chronic hepatitis B who had severe and repeated hypoglycemia due to NICTH and in whom steroid therapy appeared to be effective for the prevention of hypoglycemia.

\section{Case Report}

A 65-year-old man had suffered from chronic hepatitis B, which he had for about 40 years although the cause of infection for hepatitis B virus (HBV) was unknown. He had undergone regular medical checkups in the local clinic, and in 1993 he was introduced to the Gastroenterology Department in our hospital by the clinic because of continuous treatment. On the first visit, moderate liver dysfunction (aspartate transaminase 

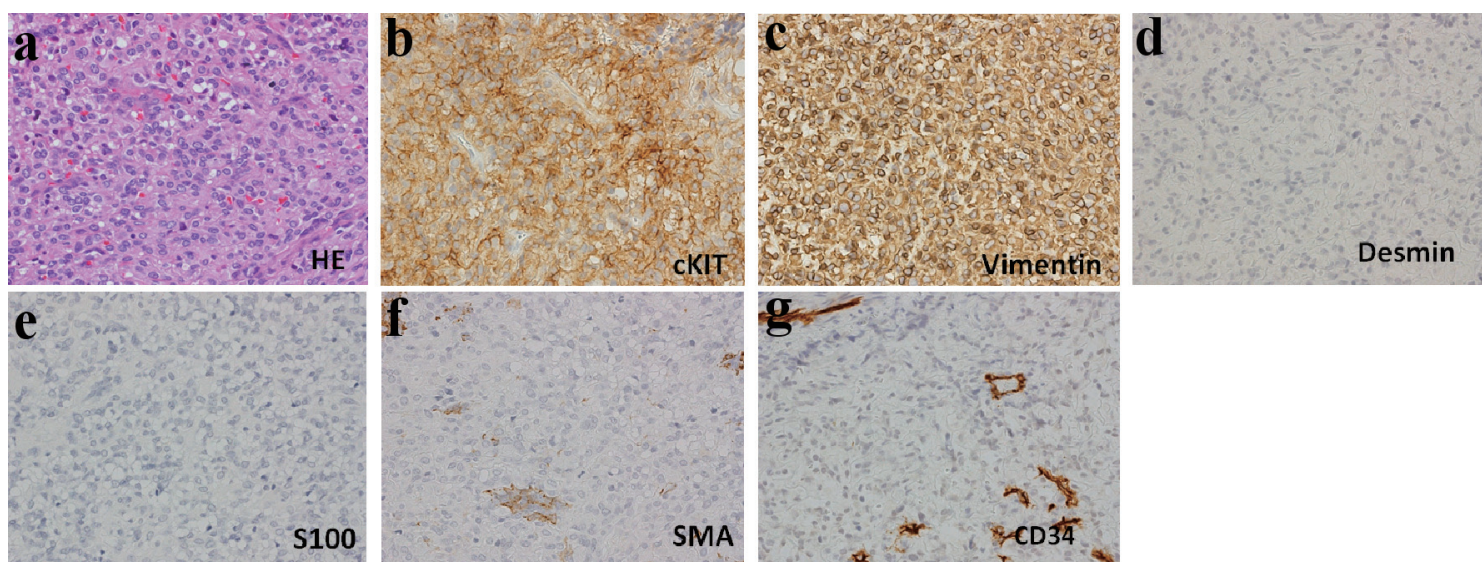

Figure 1. Histological findings in surgically resected tissues $(\times 400)$. (a) Hematoxylin and eosin (H\&E) staining: H\&E staining shows irregular proliferation of oval, partially spindle cells. Immunohistochemical staining for c-kit (CD117) (b), vimentin (c), desmin (d), S-100 (e), smooth muscle actin (SMA) (f), CD34 (g): positive findings for c-kit and vimentin.

(AST) $113 \mathrm{U} / \mathrm{L}$, alanine transaminase (ALT) $99 \mathrm{U} / \mathrm{L}$, gammaglutamyl transpeptidase (GGT) $39 \mathrm{U} / \mathrm{L}$ ) was noted. HBsAg, $\mathrm{HBe} A g$ and polymerase chain reaction for HBV (HBV-PCR) were positive. The findings in abdominal ultrasonography and computed tomography (CT) suggested liver cirrhosis. Regarding treatment, the administration of polyene phosphatidylcholine was initiated, and subsequently ursodeoxycholic acid was also added. In 2006, antiviral therapy by lamivudine (100 mg/ day) was started, resulting in the negative findings of HBVPCR after several months. In 2010, lamivudine was changed to entecavir $(0.5 \mathrm{mg} /$ day $)$, which has generally stronger antiviral effect for $\mathrm{HBV}$ than lamivudine.

In 2007, he developed massive GIST in the ileum, and immediately underwent partial resection of the tumor. Thereafter, although no obvious increase of the tumor was observed, in July in 2009, multiple tumors were found in the pelvic cavity, which strongly suggested the recurrence of GIST, and therefore, oral administration of imatinib mesylate (a tyrosine kinase inhibitor) $400 \mathrm{mg} /$ day was started. After the administration of imatinib mesylate, partial remission was obtained; but because ascites retention and bilateral edema in lower legs gradually appeared since about June 2012, imatinib mesylate was changed to sunitinib malate, another tyrosine kinase inhibitor. However, because of severe and continuous digestive symptoms due to the sunitinib malate, the sunitinib malate was changed back to imatinib mesylate after about 3 months. In February 2013, bilateral large inguinal tumors were found, and operation was performed. However, strong adhesion to the surrounding tissues prevented complete removal of the tumors from this region, resulting in partial resection. In the tissue specimens, hematoxylin and eosin (H\&E) staining showed irregular proliferation of oval, and partially spindle tumor cells, which was basically compatible with the findings of GIST cells (Fig. 1a). Immunohistochemical staining showed positive findings for c-kit protein (CD117) and vimentin, and negative findings for desmin, S-100 protein, smooth muscle actin (SMA) and CD34. These staining findings are also presented in Figure 1b-g. In addition, gene mutation analysis for c-kit showed exon 11 v599D (substitution-missense), 1676T $>$ A (substitution). Despite the negative finding for CD34, based on comprehensive histological findings, the diagnosis of peritoneal metastasis of GIST was made.

In January 2013 (i.e., just before the discovery of the bilateral large inguinal tumors), the patient noticed hypoglycemiclike symptoms, i.e., cold sweat, palpitation, and hunger sensation. During the hospitalization for operation on the bilateral large inguinal tumors in February 2013 in the Surgical Department, the patient experienced frequent apparent episodes of hypoglycemia, which required prompt oral glucose liquid intake each time. Although the exact cause of the repeated hypoglycemia during the hospitalization period was not known, the decrease of glucose release in the liver due to liver cirrhosis was suspected as one of the possible causes. Upon discharge from the hospital after surgical treatment, he was advised to increase the number of meals to avoid the potential for hypoglycemia. However, because the occasional hypoglycemic attacks continued and were increasing gradually, in mid-March 2013, he visited our outpatient department (i.e., diabetes and endocrinology) for careful examination of the cause of the hypoglycemia. After approximately 1 week after the visit, he was found unconscious by his family on the morning at home. Therefore, the patient was immediately transferred to our hospital by an ambulance. Upon arrival, simple glucose measurement revealed values below the measurement sensitivity, and thus he was admitted for emergency treatment.

Upon admission, his height and body weight were 163.0 $\mathrm{cm}$ and $54.6 \mathrm{~kg}$, respectively. The blood pressure and pulse rate were $110 / 76 \mathrm{~mm} \mathrm{Hg}$ and 110/min, respectively. He was unconscious at the time of arrival. Anemia of the palpebral conjunctiva was apparent. No abnormal chest and heart sounds were noticed. Mild distention and slight tumor-palpating of the abdomen was found, while there was no obvious tumor-palpating for the liver and spleen. Remarkable bilateral pitting edema was noted in the lower legs and dorsum of the foot. An elastic, hard tumor was palpated in the left inguinal region. There were no neurological findings. Blood tests showed liver dysfunction, mildly elevated ammonia levels, mildly decreased platelet levels that were compatible with liver cirrhosis. Mild elevation of white blood cells and apparent decrease of hemo- 
Table 1. Hematological and Blood Chemistry Tests (Mid-March, 2013 on Admission)

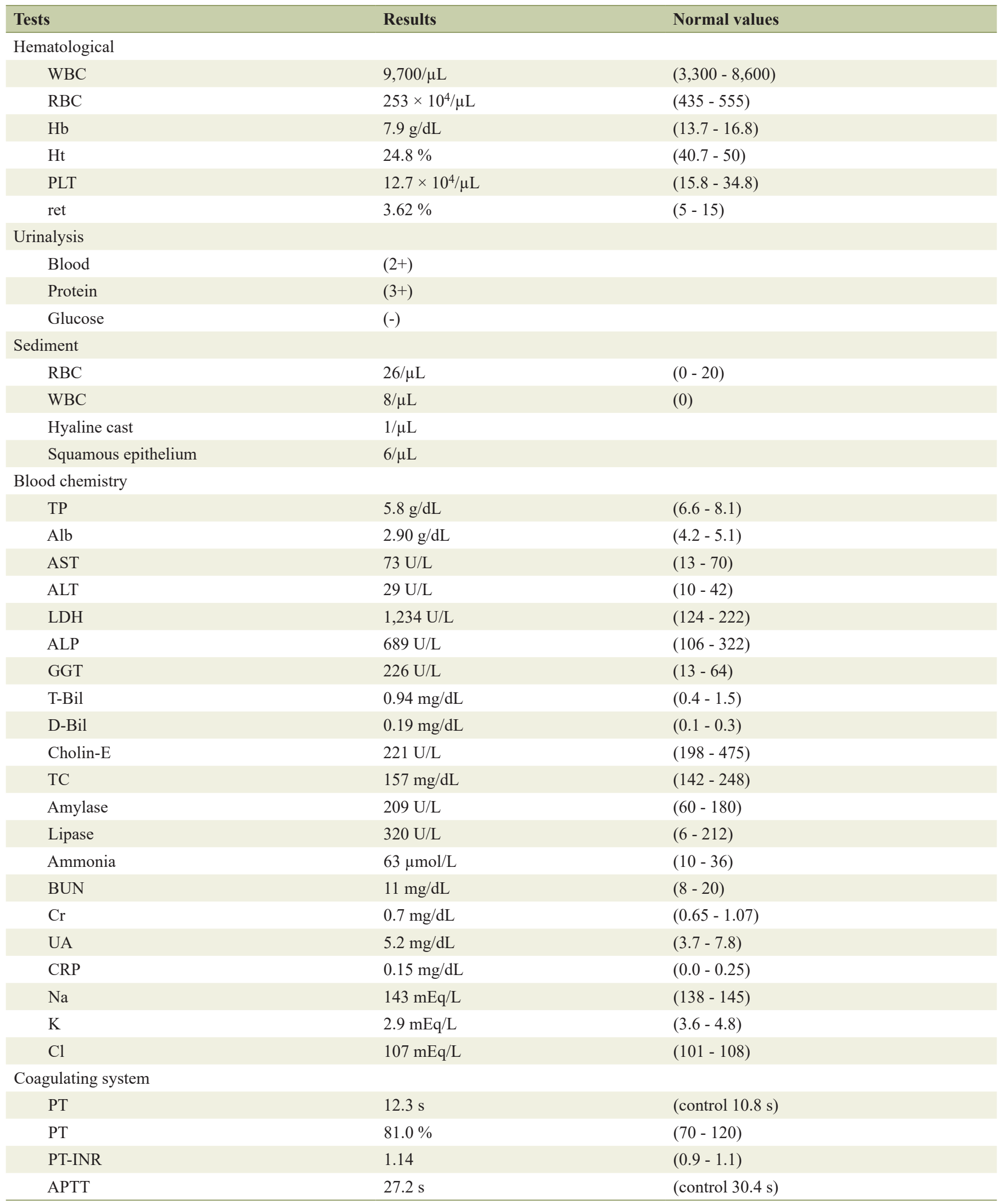


Table 1. Hematological and Blood Chemistry Tests (Mid-March, 2013 on Admission) - (continued)

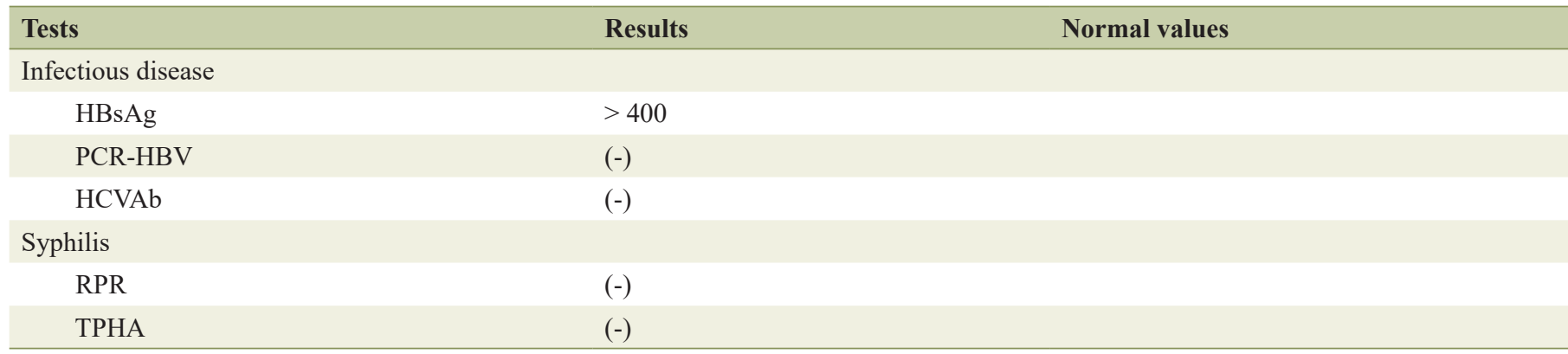

WBC: white blood cell count; RBC: red blood cell count; Hb: hemoglobin;, PLT: platelet; ret: reticulated PLT; TP: total protein; Alb: albumin; AST: aspartate transaminase; ALT: alanine transaminase; ALP: alkaline phosphatase; LDH: lactate dehydrogenase; GGT: gamma-glutamyl transpeptidase; T-Bil: total bilirubin; D-Bil: direct bilirubin; Cholin-E: cholinesterase; TC: total cholesterol; BUN: blood urea nitrogen; Cr: creatinine; UA: uric acid; CRP: C-reactive protein; Na: sodium; K: potassium; Cl: chlorine; PT: prothrombin time; PT-INR: PT-international normalized ratio; APTT: activated partial thromboplastin time; PCR-HBV: polymerase chain reaction-hepatitis B virus; HCVAb: hepatitis C virus antibody; RPR: rapid plasma regain; TPHA: Treponema pallidum hemagglutination test.

globin levels were also noted. In addition, mild elevation of amylase and lipase was observed. Although there was no apparent renal dysfunction from blood tests, proteinuria and uric blood were found in urinalysis. Hypokalemia was also noted. These findings are presented in Table 1. In endocrinological tests of fasting blood in the morning, mild elevation of thyroid stimulating hormone (TSH) was observed. Growth hormone $(\mathrm{GH})$, measured twice on another day, was mildly elevated, while IGF-1 levels were decreased. Adrenocorticotropic hormone (ACTH) and cortisol levels were normal. In diabetesrelated blood tests, extremely decreased glucose and insulin, and C-peptide levels were noted. Furthermore, in the glucagon loading test, extremely suppressed C-peptide levels were observed. Anti-insulin antibody was negative. In testing for infectious disease in the blood, HBsAg was positive $(>400)$. However, HBV-PCR was negative, which was likely due to the continuous administration of entecavir for HBV hepatitis. These findings are presented in detail in Table 2. In the imaging study, while there were no findings of pneumonia, slight bilateral pleural effusions were observed on chest X-ray. There were no abnormal findings in brain CT. In abdominal enhanced CT, massive ascites, intestinal edema, and non-enhanced multiple large and small tumors were observed (Fig. 2).

Just after admission, intravenous glucose administration immediately resulted in prompt and complete recovery of consciousness; however, severe repeated episodes of hypoglycemia (to near $20-30 \mathrm{mg} / \mathrm{dL}$ of glucose levels), especially between meals, occurred even when he took meals of $2,000 \mathrm{kcal} /$ day accompanied with the occasional addition of 200 - $300 \mathrm{kcal}$ to each meal. Therefore, continuous intravenous glucose administration ( $10 \%$ glucose $1,000 \mathrm{~mL} /$ day $)$ was initiated, but the hypoglycemia was not completely prevented. Because of the repeated and severe hypoglycemia, NICTH due to GIST was suspected, in addition to at least partial involvement of liver cirrhosis for the hypoglycemia. Therefore, we performed additional immunohistochemical staining with an anti-IGF-2 antibody (anti-IGF2 antibody: ab9574, Abcam Ltd, Cambridge, UK) using frozen surgical resection tissues, and showed that the staining was positive (Fig. 3). This strongly suggested NICTH associated with GIST producing IGF-2. To prevent severe hypoglycemia, based on previous report for treatment in NICTH [20], we started oral corticosteroid therapy (prednisolone $30 \mathrm{mg}$ /day) in the morning on the eighth day after admission. After starting prednisolone administration, immediate improvement of blood glucose level was achieved both before and after meals (from 40 - 70 to $100-180 \mathrm{mg} / \mathrm{dL}$ before meals and from 30 - 90 to 130 - $190 \mathrm{mg} / \mathrm{dL}$ after meals).

However, on the afternoon of day 9, acute aggravation of the respiratory condition emerged with findings of remarkable cardiomegaly on chest X-ray. Acute congestive heart failure was suspected based on the clinical findings. Despite increasing the dose of diuretic drugs and continuous intravenous administration of nitroglycerin with adequate respiratory management, the conditions worsened, and he died on the evening of day 9. Although the cause of acute congestive heart failure was unknown, impairment of blood perfusion in the systemic circulation due to oppression from massive GIST was considered most likely.

\section{Discussion}

In our case, because the GIST in the ileum had already been diagnosed upon the pelvic tumor, for the large inguinal tumors that occurred during imatinib therapy, the recurrence as peritoneal metastasis of GIST was suspected. The cells structure and the findings of immunohistochemical staining (c-kit protein, vimentin: positive; desmin, S-100 protein, SMA: negative) also strongly suggested the diagnosis as recurrence of GIST, although the CD34 was negative. Gene mutation analysis for c-kit (the findings of exon 11 v599D) further supported the diagnosis.

Notably, in this case, the repeated hypoglycemia attacks continued even after the partial resection of inguinal tumors, and we first suspected the hypoglycemia as a consequence of liver cirrhosis due to chronic hepatitis B. It is known that liver cirrhosis can cause insulin resistance, which is involved in impaired glucose tolerance [21]. On the other hand, liver cirrhosis could also be related to the hypoglycemia because the liver plays a central role in the maintenance of blood glucose levels 
Table 2. Endocrinological and Diabetes-Related Tests (Mid-March, 2013 on Admission)

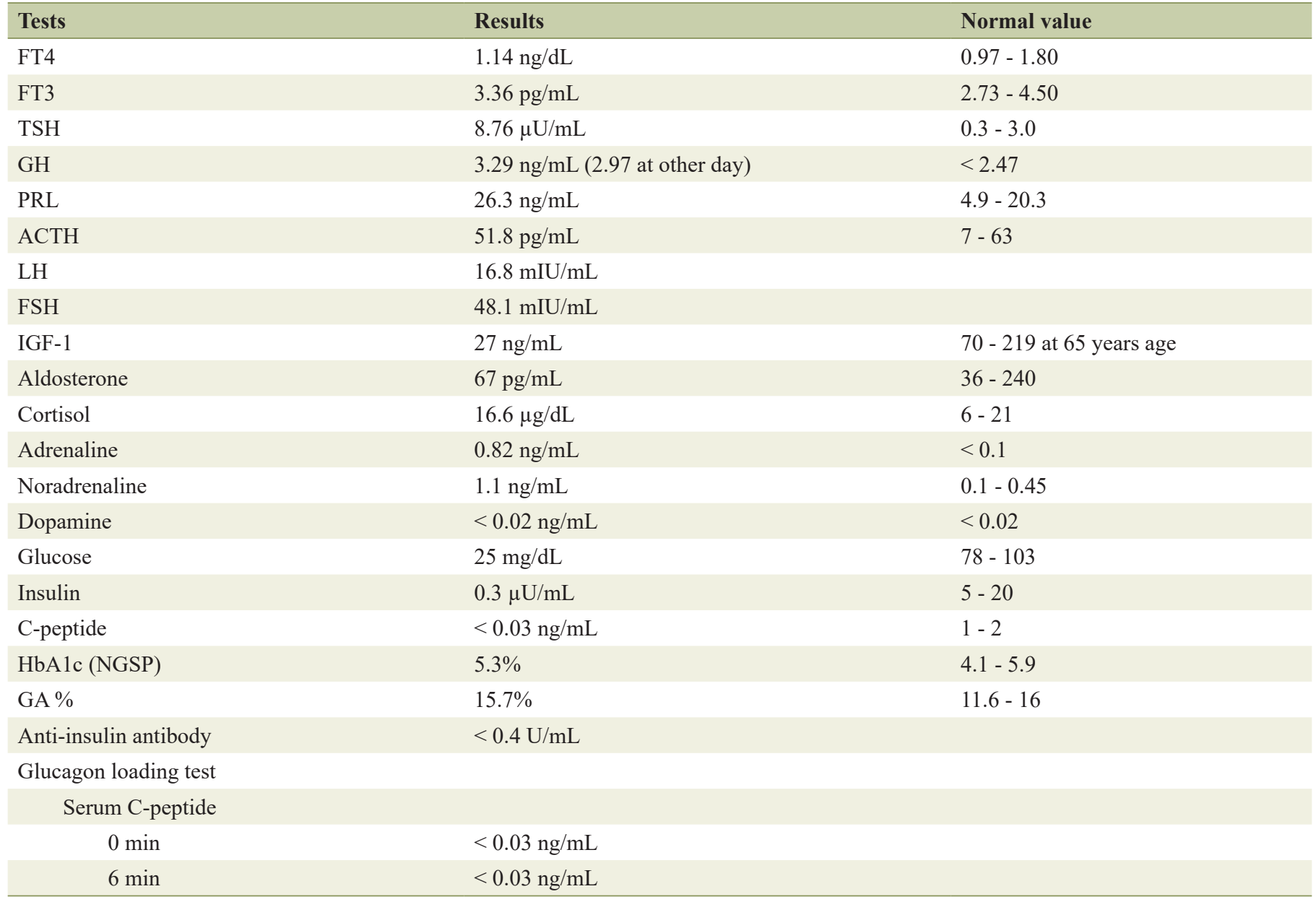

FT4: free T4; FT3: free T3; TSH: thyroid stimulating hormone; GH: growth hormone; PRL: prolactin; ACTH: adrenocorticotropic hormone; LH: luteinizing hormone; FSH: follicle stimulating hormone; IGF-1: insulin-like growth factor-1; HbA1c: hemoglobin A1c; GA: glycoalbumin.

[17-19]. However, the severity of the hypoglycemia also suggests the involvement of another mechanism, apart from the influence of liver cirrhosis. Hypoglycemia associated with adrenal cortex deficiency was unlikely because the basic values of

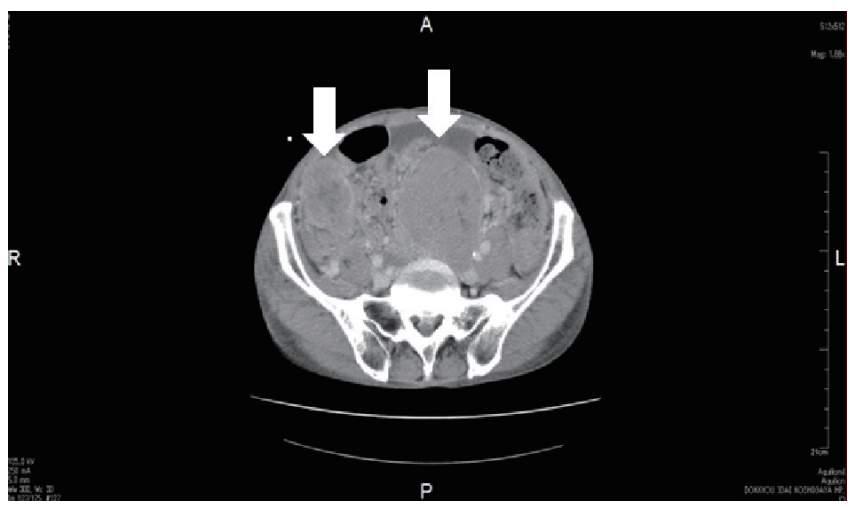

Figure 2. Enhanced abdominal computed tomography (CT). Non-enhanced multiple large and small tumors were found in the pelvic cavity on CT. Arrows show large tumors.
ACTH and cortisol were normal. In our case, elevation of TSH was also noted, although the exact reason was unknown. It is unlikely that hypothyroid influenced the hypoglycemia in our case because the hypothyroid was latent. Therefore, we next suspected NICTH due to GIST, although GIST related-NICTH is very rare [13-16]. As expected, additional immunohistochemical staining with an anti-IGF-2 antibody of the resected tumor tissues was positive, which strongly suggested GIST related-NICTH. In our case, we could not measure circulating IGF-2 levels because there was no commercial measurement approach available. While circulating IGF-2 is not necessarily elevated in all NICTH patients [5, 14], the IGF-2/IGF-1 ratio would be helpful in the diagnosis of NICTH [5]. In addition, we also could not confirm the existence of big-IGF-2 which is essential for NICTH, through Western blotting; we tried to measure IGF-2 by Western blotting in other institution during several years, but as a result we could not obtain the data. However, based on the clinical symptom of the repeated severe hypoglycemia and the positive finding of immunohistochemical staining by anti-IGF-2 antibody, we believe that the diagnosis of NICTH is definitive in this case. In addition, the hypokalemia 


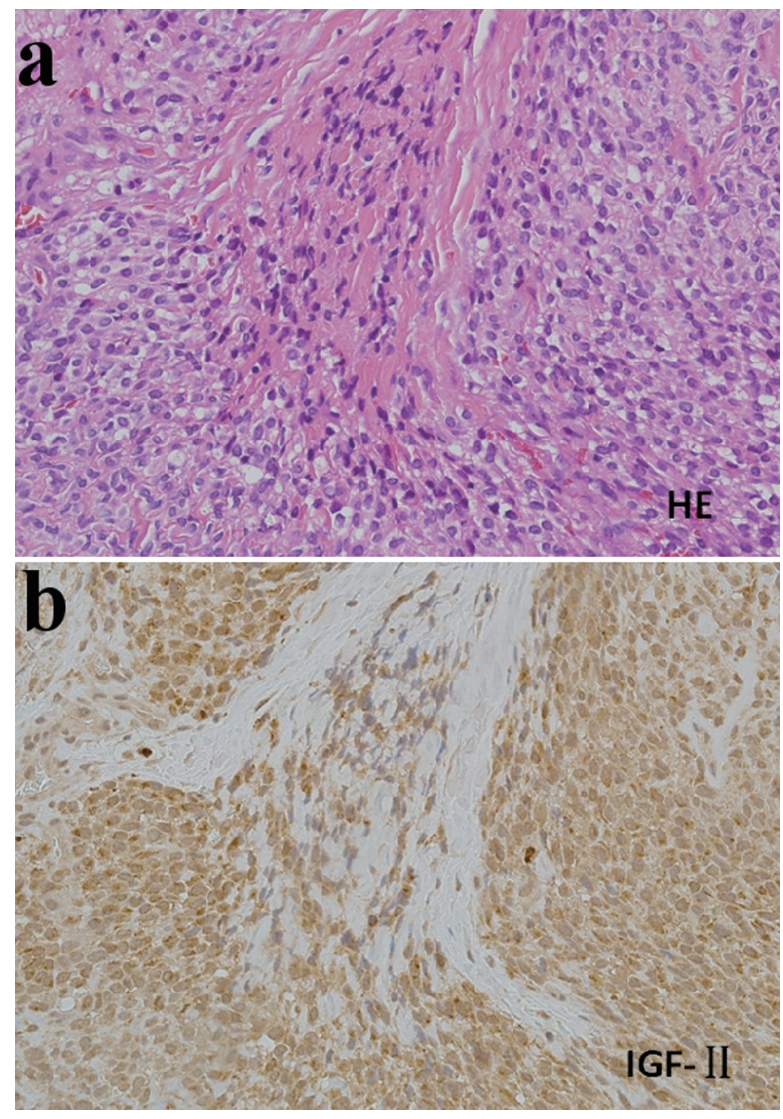

Figure 3. Histological findings in surgically resected tissues $(\times 200)$. (a) Hematoxylin-eosin (HE) staining. (b) Immunohistochemical staining with an anti-insulin-like growth factor-2 (IGF2) antibody (Anti-IGF2 antibody: ab9574, Abcam Ltd, Cambridge, UK). Cells were strongly stained with the anti-IGF2 antibody.

noticed upon admission appears not to contradict the findings of NICTH, because hypokalemia is often observed in patients with NICTH; likely due to the effect of big IGF-2 [5, 22]. Importantly, our patient had liver cirrhosis as a consequence of hepatitis $\mathrm{B}$, which is a high risk for hepatocellular carcinoma. It is known that the most common tumor related to NITCH is hepatocellular carcinoma [23]. However, in our case, there was no obvious sign of a tumor in the liver with abdominal CT.

It has been reported that steroid therapy is beneficial for the prevention of hypoglycemia by NICTH $[5,13,14,20]$. We decided to administer the steroid despite the fact that our patient had chronic hepatitis $\mathrm{B}$, for which steroid could affect the reactivation, because the HBV-PCR had been inhibited to the value below the detection limit likely due to continuous entecavir therapy. The steroid therapy with prednisolone (30 mg/ day) was very effective, which was consistent with previous reports. The treatment finding in our case may be important because it was considered that the patient in our case may have had a high risk of hypoglycemia due to the complicated liver cirrhosis, suggesting that steroid therapy for GIST might be beneficial even for such patients.

Notably, in our case, circulating fasting plasma GH level in the morning (measured twice on the other day) was not inhib- ited; the level was slightly higher than baseline value. On the other hand, IGF-1 levels, as well as glucose, insulin and C-peptide levels were inhibited, which was compatible with findings in NICTH. This was unique finding as IGF-2 inhibits GH and therefore patients with NICTH generally exhibit decreased GH $[5,6]$. The exact reason for the mildly elevated GH in our case is unknown. One of the possible reasons is counter regulatory response to the severe hypoglycemia because it is known that GH is counter hormone for hypoglycemia as well as other hormones such as adrenalin, noradrenalin and cortisol; these hormones in addition to GH also were not at least inhibited in this case. However GH in the patient with NICTH was largely inhibited at the time when the severe hypoglycemia occurred in previous reports $[14,16]$. We consider that the complication of acromegaly as the cause of the elevation of $\mathrm{GH}$ is improbable because of the lack of clinical findings, such as characteristic facial configuration and swelling of the fingers; although we missed the opportunity to use pituitary magnetic resonance imaging (MRI).

In conclusion, we treated a patient with NICTH by ileum-originating GIST and also liver cirrhosis due to chronic hepatitis B. Notably, for this case, basic GH levels were mildly elevated. Steroid therapy was effective despite the complication of liver cirrhosis, which may lead to a high risk for hypoglycemia.

\section{Acknowledgments}

None to declare.

\section{Financial Disclosure}

None to declare.

\section{Conflict of Interest}

None to declare.

\section{Informed Consent}

Not applicable.

\section{Author Contributions}

KT examined the patient and wrote the manuscript, SF, TO, MK, AU, MY, HS, KH, TT, TI, and KH reviewed the manuscript. YO performed pathological diagnosis and staining of resected tissues.

\section{Data Availability}

The authors declare that data supporting the findings of this report are available within the article. 


\section{References}

1. Nadler WH, Wolfer JA. Hepatogenic hypoglycemia associated with primary liver cell carcinoma. Arch Intern Med. 1929;44:700-710.

2. Marks V, Teale JD. Tumors producing hypoglycemia. Diabetes Metab Rev. 1991;7(2):79-91.

3. Cryer P. In: Melned S, Polonsky K, Braverman I, Kronenberg H. editors. Wiliams Text book of endocrinology. 12th ed. Philadelphia, PA: Elsevier. 2001; p. 1568.

4. De Groot JW, Rikhof B, van Doom J, Bilo Hj, Alleman MA, Honkoop AH, van der Graaf WT. Non-islet cell tumor-induced hypoglycemia: a review of the literature inducing two cases. Endocr Relat Cancer. 2007;14(4):979993.

5. Bodnar TW, Acevedo MJ, Pietropaolo M. Management of non-islet-cell tumor hypoglycemia: a clinical review. J Clin Endocrinol Metab. 2014;99(3):713-722.

6. Zachariah S, Brackenridge A, Shojaee-Moradie F, Camuncho-Hubner C, Umpleby AM, Russell-Jones D. The mechanism of non-islet cell hypoglycaemia caused by tumour-produced IGF-II. Clin Endocrinol (Oxf). 2007;67(4):637-638.

7. Dynkevich Y, Rother KI, Whitford I, Qureshi S, Galiveeti S, Szulc AL, Danoff A, et al. Tumors, IGF2, and hypoglycemia: insights from the clinic, the laboratory, and the historical archive. Endocr Rev. 2013;34(6):798-826.

8. Akahoshi K, Oya M, Koga T, Shiratsuchi Y. Current clinical management of gastrointestinal stromal tumor. World J Gastroenterol. 2018;24(26):2806-2817.

9. Chan KH, Chan CW, Chow WH, Kwan WK, Kong CK, Mak KF, Leung MY, et al. Gastrointestinal stromal tumors in a cohort of Chinese patients in Hong Kong. World J Gastroenterol. 2006;12(14):2223-2228.

10. DeMatteo RP, Lewis JJ, Leung D, Mudan SS, Woodruff JM, Brennan MF. Two hundred gastrointestinal stromal tumors: recurrence patterns and prognostic factors for survival. Ann Surg. 2000;231(1):51-58.

11. Kindblom LG, Remotti HE, Aldenborg F, Meis-Kindblom JM. Gastrointestinal pacemaker cell tumor (GIPACT): gastrointestinal stromal tumors show phenotypic characteristics of the interstitial cells of Cajal. Am J Pathol. 1998;152(5):1259-1269.

12. Hirota S, Isozaki K, Moriyama Y, Hashimoto K, Nishida T, Ishiguro S, Kawano K, et al. Gain-of-function mutations of c-kit in human gastrointestinal stromal tumors. Science. 1998;279(5350):577-580.
13. Dimitriadis GK, Gopalakrishnan K, Rao R, Grammatopoulos DK, Randeva HS, Weickert MO, Murthy N. Severe paraneoplastic hypoglycemia secondary to a gastrointestinal stromal tumour masquerading as a stroke. Endocrinol Diabetes Metab Case Rep. 2015;2015:150062.

14. Hirai H, Ogata E, Ohki S, Fukuda I, Tanaka M, Watanabe T, Satoh H. Hypoglycemia associated with a gastrointestinal stromal tumor producing high-molecular-weight insulin growth factor II: a case report and literature review. Intern Med. 2016;55(10):1309-1314.

15. Wilson JM, Ginsberg J, Cutts K, Urban S. A case of nonislet cell tumor hypoglycemia (NICTH) associated with gastrointestinal stromal tumor (GIST). Am J Case Rep. 2017; 18:984-988.

16. Yamasaki H, Itawaki A, Morita M, Miyake H, Yamamoto $\mathrm{M}$, Sonoyama H, Tanaka S, et al. A case of insulin-like growth factor 2-producing gastrointestinal stromal tumor with severe hypoglycemia. BMC Endocr Disord. 2020;20(1):60.

17. Huang Z, Sjoholm A. Ethanol acutely stimulates islet blood flow, amplifies insulin secretion, and induces hypoglycemia via nitric oxide and vagally mediated mechanisms. Endocrinology. 2008;149(1):232-236.

18. Pfortmueller CA, Wiemann C, Funk GC, Leichtle AB, Fiedler GM, Exadaktylos AK, Lindner G. Hypoglycemia is associated with increased mortality in patients with acute decompensated liver cirrhosis. J Crit Care. 2014;29(2):316 e317-312.

19. Singh D, Memon HNA, Shaikh TZ, Shah SZA. Hypoglycemia; patients with liver cirrhosis. Professional Med J. 2015;22(4):408-413.

20. Tsuro K, Kojima H, Okamoto S, Yoshiji H, Fujimoto M, Uemura M, Yoshikawa M, et al. Glucocorticoid therapy ameliorated hypoglycemia in insulin-like growth factor-II-producing solitary fibrous tumor. Intern Med. 2006;45(8):525-529.

21. Shankar TP, Solomon SS, Duckworth WC, Himmelstein S, Gray S, Jerkins T, Bobal MA, et al. Studies of glucose intolerance in cirrhosis of the liver. J Lab Clin Med. 1983;102(4):459-469.

22. Li Z, Wang J, Zhu Q, Li H, Chen Y, Chen L. Huge solitary fibrous tumor of the pleura with hypoglycemia and hypokalemia: a case report. Ann Thorac Cardiovasc Surg. 2014;20(2):165-168.

23. Garla V, Sonani H, Palabindala V, Gomez-Sanchez C, Subauste J, Lien LF. Non-islet cell hypoglycemia: case series and review of the literature. Front Endocrinol (Lausanne). 2019;10:316. 